\title{
Josephson coupling and plasma resonance in vortex crystal
}

\author{
A. E. Koshelev ${ }^{a}$ and L. N. Bulaevskii ${ }^{b *}$ \\ ${ }^{a}$ Materials Science Division, Argonne National Laboratory, Argonne, IL 60439 \\ ${ }^{\mathrm{b}}$ Los Alamos National Laboratory, Los Alamos, NM 87545
}

We investigate the magnetic field dependence of the plasma resonance frequency in vortex crystal state. We find that low magnetic field induces a small correction to the plasma frequency proportional to the field. The slope of this linear field dependence is directly related to the average distance between the pancake vortices in the neighboring layers, the wandering length. This length is determined by both Josephson and magnetic couplings between layers. At higher fields the plasma frequency is suppressed collectively and is determined by elastic energy of the vortex lattice. Analyzing experimental data, we find that (i) the wandering length becomes comparable with the London penetration depth near $\mathrm{T}_{c}$, (ii) at small melting fields $(<20 \mathrm{G})$ the wandering length does not change noticeably at the melting transition demonstrating existence of the line liquid phase in this field range, and (iii) the self consistent theory of pancake fluctuations describes very well the field dependence of the Josephson plasma resonance frequency up to the melting point.

\section{Introduction}

Josephson coupling characterizes the ability of layered superconductors to carry supercurrents across the layers. In very anisotropic superconductors this coupling is suppressed by magnetic field applied along the c-axis. Thermal fluctuations and uncorrelated pinning lead to misalignment of pancake vortices induced by the magnetic field [1] (see Fig. 11). Misalignment results in nonzero phase difference and in the suppression of the Josephson interlayer coupling. This suppression is quantitatively characterized by the "local coherence factor" $\mathcal{C} \equiv\left\langle\cos \varphi_{n, n+1}(\mathbf{r})\right\rangle$, where $\varphi_{n, n+1}(\mathbf{r})$ is the gauge-invariant phase difference between layers $n$ and $n+1,\langle\ldots\rangle$ means average over thermal disorder and pinning. Josephson plasma resonance (JPR) measurements in highly anisotropic layered superconductors [2] probe directly the interlayer Josephson coupling and the effect of pancake vortices on this coupling, because the squared JPR frequency, $\omega_{p}^{2}$, in the most part of vortex phase diagram, is proportional to

\footnotetext{
*This work was supported by the NSF Office of the Science and Technology Center under contract No. DMR-9120000 and by the U. S. DOE, BES-Materials Sciences, under contract No. W-31- 109-ENG-38. Work in Los Alamos is supported by U.S. the DOE.
}

the average interlayer Josephson energy [3],

$$
\omega_{p}^{2} \approx \omega_{0}^{2} \mathcal{C} \propto J_{0} \mathcal{C}
$$

where $\omega_{0}(T)=c / \sqrt{\epsilon_{0}} \lambda_{c}(T)$ is the zero field plasma frequency, $\lambda_{c}(T)$ is the c-component of the London penetration depth, $\epsilon_{0}$ is dielectric constant, and $J_{0}$ is the Josephson critical current.

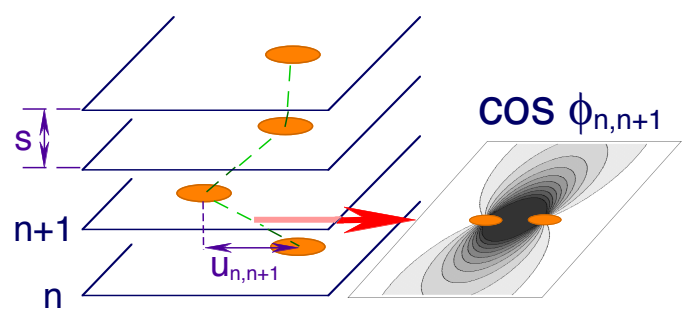

Figure 1. Meandering of pancakes along the vortex line in layered superconductors and grayscale plot of $\cos \phi_{n, n+1}$ near two misaligned pancakes.

The JPR measurements performed in the liquid vortex phase at relatively high magnetic fields, $B>B_{J}=\Phi_{0} / \lambda_{J}^{2}$, revealed that the plasma frequency drops approximately as $1 / \sqrt{B}$ [2], where $\lambda_{J}=\gamma s$ is the Josephson length, $\gamma$ is the anisotropy ratio and $s$ is the interlayer distance. The above dependence is characteristic for the pancake liquid weakly correlated along the $c$ 
axis. [1] In this phase many pancake vortices contribute to the suppression of the phase difference at a given point. In contrast, in the vortex solid pancake vortices form aligned stacks and suppression of coupling is caused by weak misalignments of the pancake vortices due to the thermal fluctuations and random pinning. JPR measurements in $\mathrm{Bi}_{2} \mathrm{Sr}_{2} \mathrm{CaCu}_{2} \mathrm{O}_{8-\delta}(\mathrm{Bi}-2212)$ crystals [5,6] have shown that the JPR frequency decreases approximately linearly with field in the vortex solid. In the fields above 20 Oe the interlayer phase coherence changes drastically at the transition line, implying the decoupling nature of the first-order melting transition in agreement with theoretical expectations (see, e.g., Ref. [7]). On the other hand, at smaller field phase coherence does not change considerably at the melting point. [5]

In this paper we consider the Josephson coupling and JPR in the vortex lattice. We focus on the suppression of coupling due to thermal fluctuations of pancake vortices near the equilibrium crystal positions, and neglect influence of pinning potential. For real Bi-2212 crystals this approximation is justified at sufficiently high temperatures ( $\gtrsim 40 \mathrm{~K})$. This problem has been considered in the past in the simple limiting cases. [1. 84 However quantitative calculation suitable for comparison with existing JPR data [5.6] in a wide field range is still absent. At small fields, when vortices act independently, $\omega_{p}^{2}$ decreases linear with $B$. The linear dependence was observed experimentally in Refs. [0.6] in solid state in Bi-2212 crystals. In fields below 20 Oe near $T_{c}$ this linear dependence extends to the liquid state providing evidence for a line structure of the vortices in the liquid at low fields. The regime of independent vortices has been considered in Ref. [8]. In this paper we extend our consideration to higher fields up to the melting field.

\section{Low fields. Isolated vortex lines}

Consider small magnetic fields $B \ll B_{J}, B_{\lambda} \equiv$ $\Phi_{0} / 4 \pi \lambda_{a b}^{2}$. At these fields regions of suppressed coupling are localized near the vortex lines (pancake stacks) and do not overlap (single vortex regime) [8]. The field-induced change in $\mathcal{C}$ in this regime is given by $\delta \mathcal{C} \equiv 1-\mathcal{C}=B I / \Phi_{0}$, where $I=\int d^{2} \mathbf{r}\left(1-\cos \left(\varphi_{n, n+1}(\mathbf{r})\right)\right)$, and $\varphi_{n, n+1}(\mathbf{r})$ is phase difference induced by fluctuation displacements $\mathbf{u}_{n}$ in a single line. The same integral determines the tilt stiffness due to the Josephson coupling. We split integration domain in $I$ into two region, $r<R$ and $r>R$, where $R$ is the intermediate scale $r_{w}<R<\gamma s$, with $r_{w}^{2} \equiv\left\langle\mathbf{u}_{n, n+1}^{2}\right\rangle$ and $\mathbf{u}_{n, n+1} \equiv \mathbf{u}_{n+1}-\mathbf{u}_{n}$. At $r<R$ we can neglect screening due to the Josephson currents and take

$$
\varphi_{n, n+1}(\mathbf{r})=\arctan \frac{y-u_{y n+1}}{x-u_{x n+1}}-\arctan \frac{y-u_{y n}}{x-u_{x n}} .
$$

At $r>R$ we can take $\varphi_{n, n+1}$ in linear approximation with respect to $\mathbf{u}_{n}$,

$\varphi_{n, n+1}\left(\mathbf{r}, k_{z}\right) \approx-\int \frac{s d k_{z}}{2 \pi} \tilde{k}_{z}\left[\mathbf{u}\left(k_{z}\right) \times \nabla\right] \mathrm{K}_{0}\left(\frac{\tilde{k}_{z} r}{\gamma}\right)$,

where $\mathbf{u}\left(k_{z}\right)=s \sum_{n} \exp \left(-i s k_{z} n\right) \mathbf{u}_{n}, \quad \tilde{k}_{z} \equiv$ $(2 / s) \sin \left(s k_{z} / 2\right)$, and $\mathrm{K}_{0}(z)$ is the modified Bessel function. At intermediate distances $r_{w} \ll r \ll$ $\lambda_{J}$, both expressions give the same simple result $\varphi_{n, n+1}(\mathbf{r}) \approx\left[\mathbf{r} \times \mathbf{u}_{n, n+1}\right] / r^{2}$. Using above asymptotics of $\varphi_{n, n+1}$ we obtain

$$
\begin{aligned}
I & =\frac{\pi}{2} \int \frac{d k_{z}}{2 \pi}\left(1-\cos \left(s k_{z}\right)\right)\left|u\left(k_{z}\right)\right|^{2} \\
& \times \ln \left(\frac{3.72 \lambda_{J}^{2}}{u_{n, n+1}^{2}\left(1-\cos \left(s k_{z}\right)\right)}\right) .
\end{aligned}
$$

Weak logarithmic dependence on $u_{n, n+1}$ leads to the nonharmonic tilt energy. [9] In the following we will eliminate this nonharmonicity using the self consistent harmonic approximation (SCHA), which results in the substitution $\ln \left(A / u_{n, n+1}^{2}\right) \rightarrow$ $\ln \left(0.24 A / r_{w}^{2}\right)$. Approximate evaluation of the above integral gives a simple practical relation connecting the field-induced correction of the plasma frequency $\omega_{p}(B, T)$ with $r_{w}$ for the case $r_{w}<\lambda_{J}<a$ :

$$
\frac{\omega_{0}^{2}(T)-\omega_{p}^{2}(B, T)}{\omega_{0}^{2}(T)} \approx \frac{\pi B r_{w}^{2}}{2 \Phi_{0}} \ln \frac{0.8 \lambda_{J}}{r_{w}} .
$$

This relation allows one to extract $r_{w}^{2}$ from the plasma resonance measurements.

We now calculate $r_{w}^{2}$ when wandering of the vortex lines is caused by thermal fluctuations. In the single vortex regime $r_{w}^{2}$ is determined by the 
wandering energy consisting of the Josephson and magnetic contributions,

$$
\mathcal{F}_{w} \approx \frac{1}{2} \int \frac{d k_{z}}{2 \pi}\left[\varepsilon_{1 J} \tilde{k}_{z}^{2}+w_{M}\right]\left|u\left(k_{z}\right)\right|^{2},
$$

where $\varepsilon_{1 J} \approx\left(\varepsilon_{0} / \gamma^{2}\right) \ln \left(1.33 \gamma /\left(r_{w} \tilde{k}_{z}\right)\right)$ is the line tension due to the Josephson coupling, $w_{M} \approx$ $\left(\varepsilon_{0} / \lambda_{a b}^{2}\right) \ln \left(1.5 \lambda_{a b} / r_{w}\right)$ is the effective cage potential, which appears due to nonlocal magnetic interactions between pancake vortices in different layers (it describes the magnetic tilt stiffness at wave vectors $\left.k_{z}>1 / r_{w}\right)$ [10, ]], and $\varepsilon_{0} \equiv$ $\Phi_{0}^{2} /\left(4 \pi \lambda_{a b}\right)^{2}$. Assuming Gaussian fluctuations of pancake vortices we obtain

$$
r_{w T}^{2}=\frac{8 T}{s w_{M}} \frac{1}{1+\zeta+\sqrt{1+\zeta}}
$$

where the parameter $\zeta(T) \approx 4 \lambda_{a b}^{2}(T) / \lambda_{J}^{2}$ describes the relative roles of the Josephson and magnetic interactions. Substituting this result into Eq. (2) we obtain

$$
\frac{\omega_{0}^{2}-\omega_{p}^{2}(B)}{\omega_{0}^{2}} \approx \frac{4 \pi \lambda_{a b}^{2} B T}{s \epsilon_{0} \Phi_{0}} \frac{1}{1+\zeta+\sqrt{1+\zeta}} .
$$

This result of the single vortex regime is valid in both solid and liquid states for $B \ll B_{J}$, because in this field range wandering of lines at short scales does not change much at the melting point. Eq. (5) describes fairly well the suppression of the plasma frequency at small fields [8]. With the data of Ref. [5] for underdoped Bi2212 with $\mathrm{T}_{c} \approx 84.5 \mathrm{~K} \mathrm{Eq}$. (2) gives unexpectedly large wandering length $r_{w} \approx 1 \mu \mathrm{m}$ at $77 \mathrm{~K}$, which is comparable with both $\lambda_{a b}$ and $\lambda_{J}$ at this temperature. However, we found that this estimate is a in good agreement with the theoretical calculation (14).

\section{High fields, $B>B_{J}, B_{\lambda}$}

As the field increases two competing effects start to influence pancake fluctuations and field dependence of the average Josephson energy. Vortex interactions diminish pancake fluctuations. On the other hand, collective suppression of the Josephson energy decreases tilt stiffness and enhances pancake fluctuations. Using general relations connecting the phase perturbations with the elastic lattice deformations (see, e.g. Ref. [1]), we obtain

$\delta \mathcal{C}=\frac{\left\langle\left[\varphi_{n, n+1}\right]^{2}\right\rangle}{2} \approx \frac{\left(2 \pi s n_{v}\right)^{2}}{2}$
$\times \int \frac{d^{2} q d k_{z}}{(2 \pi)^{3}} \sum_{Q<q_{m}} \frac{\tilde{k}_{z}^{2}\left([\mathbf{Q} \times \mathbf{q}]^{2}\left\langle\mathbf{u}_{l}^{2}\right\rangle+\left(q^{2}+\mathbf{Q q}\right)^{2}\left\langle\mathbf{u}_{t}^{2}\right\rangle\right)}{q^{2}\left((\mathbf{q}+\mathbf{Q})^{2}+\tilde{k}_{z}^{2} / \gamma^{2}\right)^{2}}$ where $n_{v} \equiv B / \Phi_{0}, \mathbf{Q}$ are the reciprocal lattice vectors (the cut off $q_{m} \approx 2.2 / r_{w}$ in the summation over $\mathbf{Q}$ is established by comparison with the single vortex regime), and $\mathbf{u}_{l}\left(\mathbf{q}, k_{z}\right)\left(\mathbf{u}_{t}\left(\mathbf{q}, k_{z}\right)\right)$ are the longitudinal (transversal) elastic lattice displacements. Integration with respect to the in-plane wave vector $\mathbf{q}$ is limited by the first Brillouin zone, which we approximate by the circle $q<K_{0}, K_{0}^{2}=4 \pi n_{v}$. For thermal Gaussian fluctuations the mean squared averages $\left\langle\mathbf{u}_{t, l}^{2}\right\rangle \equiv$ $\left\langle\left|\mathbf{u}_{t, l}\left(\mathbf{q}, k_{z}\right)\right|^{2}\right\rangle$ are determined by the corresponding components of the elastic matrix $\Phi_{t, l}\left(\mathbf{q}, k_{z}\right)$

$$
\left\langle\left|\mathbf{u}_{t, l}\left(\mathbf{q}, k_{z}\right)\right|^{2}\right\rangle=T / \Phi_{t, l}\left(\mathbf{q}, k_{z}\right) .
$$

At high fields $B>B_{J}, B_{\lambda}$, and at large $k_{z}, k_{z} \gg$ $1 / \lambda_{a b}, \sqrt{n_{v}}$ we have

$$
\begin{aligned}
& \Phi_{t}\left(q, k_{z}\right)=C_{66} q^{2}+\Phi_{44}\left(q, k_{z}\right), \\
& \Phi_{l}\left(q, k_{z}\right)=\Phi_{11}(q)+\Phi_{44}\left(q, k_{z}\right),
\end{aligned}
$$

where $\Phi_{11} \approx \frac{B^{2}}{4 \pi}\left(1-\frac{q^{2}}{4 K_{0}^{2}}\right)$ is the compression stiffness, $C_{66}=A_{66} n_{v} \epsilon_{0} / 4$ is the shear modulus, parameter $A_{66}<1$ describes fluctuation suppression of $C_{66}$, which we approximate as $A_{66}=1-0.4 B / B_{m}$ with $B_{m}$ being the melting field, and

$$
\begin{aligned}
& \Phi_{44}\left(q, k_{z}\right) \approx \frac{n_{v} \varepsilon_{0} k_{z}^{2}}{2 \gamma^{2}} \ln \frac{0.11 a^{2}}{r_{w}^{2}\left(1-0.53 q^{2} / K_{0}^{2}\right)^{2}} \\
& +\frac{n_{v} \varepsilon_{0}}{2 \lambda_{a b}^{2}} \ln \left(0.5+\frac{0.13 a^{2}}{r_{w}^{2}}\right)+\frac{B^{2}}{4 \pi \lambda_{a b}^{2}} \frac{k_{z}^{2}}{k_{z}^{2}+\gamma^{2} q^{2}},
\end{aligned}
$$

is the tilt stiffness, computed within SCHA, with $a \equiv 1 / \sqrt{n_{v}}$. The wandering length $r_{w}$ has to be determined self-consistently from the equation

$$
\begin{aligned}
r_{w}^{2} & =2 \int \frac{d^{2} q d k_{z}}{(2 \pi)^{3}}\left(1-\cos \left(s k_{z}\right)\right) \\
& \times\left(\left\langle\left|\mathbf{u}_{l}\left(\mathbf{q}, k_{z}\right)\right|^{2}\right\rangle+\left\langle\left|\mathbf{u}_{t}\left(\mathbf{q}, k_{z}\right)\right|^{2}\right\rangle\right) .
\end{aligned}
$$


Expression (6) for $\delta \mathcal{C}$ can be naturally split into the collective contribution $\delta \mathcal{C}_{\text {coll }}$, corresponding to $Q=0$ term in the $Q$-summation,

$$
\delta \mathcal{C}_{\text {coll }} \approx \frac{\left(2 \pi s n_{v}\right)^{2}}{2} \int \frac{d^{2} q d k_{z}}{(2 \pi)^{3}} \frac{\tilde{k}_{z}^{2} q^{2}\left\langle\mathbf{u}_{t}^{2}\right\rangle}{\left(\mathbf{q}^{2}+\tilde{k}_{z}^{2} / \gamma^{2}\right)^{2}},
$$

and the local contribution $\delta \mathcal{C}_{\text {loc }}$ coming from $Q>$ 0 terms. At high fields $B \gg \Phi_{0} / \lambda_{J}^{2}$ we obtain approximate expression for $\delta \mathcal{C}_{l o c}$, which resembles the single-vortex result (2)

$$
\delta \mathcal{C}_{l o c} \approx \frac{\pi n_{v} r_{w}^{2}}{2} \ln \frac{0.58 a}{r_{w}} .
$$

If we consider suppression of coupling in the cylindrical Bravais cell near the chosen vortex line, then the local term determines suppression of coupling caused by this vortex line and the collective term determines suppression of coupling caused by all other vortex lines. In general, relative role of the collective term in $\delta \mathcal{C}$ grows with field. We use above expressions to calculate the field dependence of $\mathcal{C}$ for comparison with JPR data.

Recently detailed measurements of field dependence of JPR frequency in the vortex crystal state of Bi-2212 have been done by M. Gaifullin et al. using frequency scan. [6] To compare our calculations with JPR data we need to know $\lambda_{a b}(T)$ and $\gamma=\lambda_{c} / \lambda_{a b} . \quad \lambda_{c}$ is extracted directly from JPR frequency at $B=0, \lambda_{c}(T)=c / \sqrt{\epsilon_{0}} \omega_{0}(T)$ taking $\epsilon_{0}=11$ and $\gamma$ is chosen as a fitting parameter. Fig. 2 compares the computed dependence $\mathcal{C}(B)$ with $\left(\omega_{p}(B) / \omega_{p}(0)\right)^{2}$ for three values of temperature. We also show obtained values of $\lambda \equiv \lambda_{a b}$ and $\gamma$. We obtain $\gamma$, that slightly grows with temperature (from 460 at $40 \mathrm{~K}$ to 510 at $60 \mathrm{~K}$ ). Such enhancement of $\gamma$ is expected due to the phase fluctuations.

In conclusion, we have calculated the field dependence of the JPR frequency in the vortex crystal. In the single vortex regime at low magnetic fields the JPR provides a direct probe for meandering of individual lines. The wandering length extracted from the JPR data is in agreement with the theoretical calculations. Our theory of pancake fluctuations gives a very good description of the field dependence of the plasma frequency up to the melting field. The authors thank M. Gaifullin, Y. Matsuda, T. Tamegai, and T. Shibauchi for providing their experimental data prior publication and V. Vinokur for constructive comments.

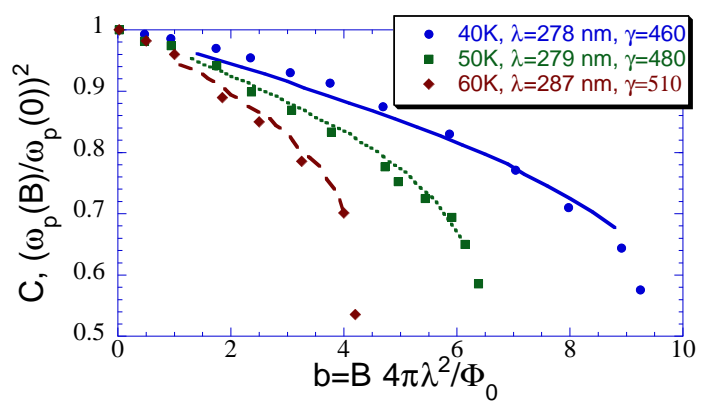

Figure 2. Comparison of the field dependence of the reduced plasma frequency squared (courtesy of M. Gaifullin and Y. Matsuda [6]) and calculated field dependence of the local coherence factor $\mathcal{C}$.

\section{REFERENCES}

1. L. I. Glazman and A. E. Koshelev, Phys. Rev. B, 43 (1991) 2835; L. Daemen et al., Phys. Rev. Lett. 70, 1167 (1993); Phys. Rev. B 47 (1993) 11291;

2. Y. Matsuda et al., Phys. Rev. Lett. 75 (1995) 4512; ibid 78 (1997) 1972; O. K. C. Tsui et al., Phys. Rev. Lett., 73 (1994) 724; ibid $\mathbf{7 6}$ (1996) 819; N. P. Ong et al., Physica C 293 (1997) 20.

3. L. N. Bulaevskii, M. P. Maley, and M. Tachiki, Phys. Rev. Lett. 74 (1995) 801.

4. A. E. Koshelev, Phys. Rev. Lett. 77 (1996) 3901; A. E. Koshelev, L. N. Bulaevskii, and M. P. Maley, Phys. Rev. Lett. 81 (1998) 9.

5. T. Shibauchi et al., Phys. Rev. Lett. 83 (1999) 1010;

6. M. Gaifullin et al., preprint.

7. G. Blatter et al., Phys. Rev. B 54 (1996) 72.

8. L. N. Bulaevskii et al., cond-mat/9907462

9. C. Krämer, Physica C 256 (1996) 236; T. R. Goldin and B. Horovitz, Phys. Rev. B 58 (1998) 9524.

10. A. E. Koshelev and P. H. Kes, Phys. Rev. B 48 (1993) 6539. 